\title{
Inhomogeneous magnetism induced in a superconductor at superconductor-ferromagnet interface
}

\author{
V. N. Krivoruchko* and E. A. Koshina \\ Donetsk Physics 83 Technology Institute, Donetsk, Ukraine \\ (*Email address: krivoruc@host.dipt.donetsk.ua)
}

(November 1, 2018)

Typeset using REVTEX 


\section{INTRODUCTION}

Ferromagnetism and superconductivity are antagonistic to each other states and ferromagnetism, being usually much stronger than superconductivity, destroys the latter. However, when dealing with superconductor-ferromagnet $(S F)$ hybrid structures the coexistence of these two phenomena is possible. If a normal ferromagnetic metal $(F)$ is in good electric contact with a superconductor $(S)$, the former can acquire some superconducting properties, however, with spatially inhomogeneous order parameter $[1,2]$. The spatial variation of the superconducting order parameter in the ferromagnet arises as a response of the Cooper pair to the energy difference between the two spin directions in ferromagnet. Due to nonzero momentum of the pairs, the usually exponentially decaying Cooper's pair density in the $F$ layer has a damped-oscillatory behavior (see, e.g., Ref. [3] for details). As has been theoretically predicted, this causes the formation of the so-called $\pi$-type superconductivity $[4,5]$, an enhancement of $d c$ Josephson current in SFIFS tunnel junctions [6,7] (where $I$ is an insulator) and the transition temperature oscillation for $S F$ multilayers [8,9] as a function of the exchange field $h_{F}$ or of the thickness $d_{F}$ of a ferromagnet. Experiments that have been performed on SFS weak links [10,11] and SIFS tunnel junctions [12] directly prove the $\pi$-phase superconductivity. Planar tunneling spectroscopy reveals also a $\pi$-phase shift in the order parameter, when superconducting correlations coexist with ferromagnetic order [13]. However, experiments on $T_{C}\left(d_{F}\right) S F$ multilayers behavior are still not so conclusive: the nonmonotonic oscillation-like behavior of $T_{C}\left(d_{F}\right)$ was also observed in ,e.g., [14 -16] while negative results were reported in, e.g., $[17,18]$. For interpretation of experimental results, along with a mechanism of $\pi$-type superconductivity and the suppression of $T_{C}$ due to a strong exchange field of a ferromagnet other mechanisms were suggested such as a "magnetically dead" interfacial layer [16], the effects of a finite interface transparency [18], spin-flip scattering $[3,19]$ and other.

The proximity induced superconductivity of the $F$ metal in $S F$ sandwiches has been intensively studied, while not so much attention has been payed to the modification of the electron spectrum of the superconductor in a region near the $S F$ interface (an exception is a nonequlibrium case, that we do not consider here). In many cases, the Curie temperature of the ferromagnet is by order of magnitude larger than the superconducting critical temperature $T_{C}$, and, in a rough approximation, for $S F$ hybrid structures the "leakage of magnetism" into the $S$ layer should be quite strong. Indeed, let us consider the proximity effects at the $S F$ bilayer interface. When diffusing into the ferromagnetic layer a superconducting electron is subject to an interaction from the local exchange field. E.g., an electron with a spin, e.g. "up", has an extra energy $H_{\text {exc }}=\mu_{B} h_{F}$ caused by the intrinsic magnetic field $h_{F}$ in ferromagnet ( $\mu_{B}$ is the Bohr magneton). After tunneling back from the $F$ to $S$ layer, one can expect a kind of tunneling interaction that is similar to the superconducting proximity effect. Actually, in the superconductor the electron quickly loses its extra energy $\delta E \simeq H_{e x c}$ during the time $\tau^{\sim} \hbar / H_{e x c}$ being related to a small range $\lambda_{F}{ }^{\sim} \hbar v_{F} / H_{e x c}(\hbar$ is Planck's constant, $v_{F}$ is Fermi velocity). Such an equilibrium process leads to a modification of the electron spectrum of the superconductor on nanoscale length $\lambda_{F}$. In most cases $\lambda_{F} \sim 10^{-8} \mathrm{~m}$, so one can assume the importance of such processes for hybrid superconductorferromagnetic systems with thin $S$ layers. However, in spite of its importance, a theoretical analysis of induced magnetic properties of the $S$ metal being in contact with the $F$ one is 
still lacking.

The investigation of a "magnetic proximity" effect in $S F$ nanostructures is the purpose of our work. On the basis of the microscopic theory for proximity coupled $S F$ bilayer (Sec. II), we calculate the spatial variation of the superconducting order parameter into the $S$ layer near the interface as a function of the ferromagnetic exchange field and boundary parameters (Sec. III A). Quasiparticle's densities of states (DOS) variation in the $S$ layer as a function of the distance from the $S F$ boundary is also considered on the same basis (Sec. III B). It is shown that the superconducting pair subjected to the proximity induced exchange field breaks, which leads to the formation of quasiparticle subbands in the superconducting energy gap. In the absence of spin-orbit (spin-flip) scattering, the subgap bands accommodate only

one spin of quasiparticle. Equilibrium local magnetization of the $S$ layer due to the induced exchange correlation is also calculated (Sec. III C). We end with the Conclusion where we emphasize the significance of the leakage of magnetism into superconductor for experiments on $S F$ structures.

\section{PROXIMITY EFFECT MODEL}

Let us consider the proximity effects in the $S F$ bilayer of a massive $S\left(d_{S} \gg \xi_{S} \gg l_{S}\right)$ and a thin $F\left(l_{F} \ll d_{F} \ll \xi_{F}\right)$ layers, for the case of an arbitrary transparency of the $S F$ boundary, the proximity effect strength and general relation between Curie temperature of the $F$ metal and superconducting critical temperature of the $S$ metal. Here $\xi_{S}=\left(\frac{D_{S}}{2 \pi T_{C}}\right)^{1 / 2}$ and $\xi_{F}=\left(\frac{D_{F}}{2 H_{e x c}}\right)^{1 / 2}$ are the effective coherence lengths; $D_{S, F}$ are the diffusion coefficients, $d_{S, F}$ are the thicknesses and $l_{S, F}$ are the electron mean free paths of the $S$ and $F$ layers, respectively; $H_{e x c}$ is the exchange energy of the ferromagnet. (Henceforth, we have taken the system of units with $\hbar=k_{B}=1$.) We assume the "dirty" limit for both metals. The superconducting critical temperature of the $F$ material equals zero. All quantities for $S$ layer are assumed to depend only on a single coordinate $x$ normal to the interface surface of the materials, while, due to the relation $d_{F} \ll \xi_{F}$ on thickness, one can neglect the coordinate dependence within the $F$ layer. We also expect the $F$ layers magnetization to be aligned parallel to the interface, so that they do not create a spontaneous magnetic flux penetrating into the $S$ layer. Under these conditions, the only magnetic interaction which can affect the superconductor is the short-range exchange interaction between the superconducting quasiparticles and magnetic moments into the ferromagnet.

As is well known, the normal and anomalous Green's functions of the "dirty" metals are described by the Usadel equations [20]. We will restrict ourselves to the case when the spin-orbit scattering is absent and spin "up" and spin "down" electron subbands do not mix with each other (see, e.g., [3]). With the assumptions given above the $S F$ bilayer is described by the following equations system for one (e.g." up") spin subband (the domain $x \geq 0$ is occupied by the $S$ metal and $x<0$ by the $F$ metal):

$$
\Phi_{S}=\Delta_{S}+\xi_{S}{ }^{2} \frac{\pi T_{C}}{\omega G_{S}}\left[G_{S}^{2} \Phi_{S}^{\prime}\right]^{\prime}, \quad G_{S}=\frac{\omega}{\left(\omega^{2}+\Phi_{S} \tilde{\Phi}_{S}\right)^{1 / 2}}
$$




$$
\begin{gathered}
\Delta_{S} \ln \left(T / T_{C}\right)+2 \pi T \sum_{\omega>0}\left[\left(\Delta_{S}-\Phi_{S} G_{S}\right) / \omega\right]=0, \\
\Phi_{F}=\xi_{F}{ }^{2} \frac{\pi T_{C}}{\tilde{\omega} G_{F}}\left[G_{F}^{2} \Phi_{F}^{\prime}\right]^{\prime}, \quad G_{F}=\frac{\tilde{\omega}}{\left(\tilde{\omega}^{2}+\Phi_{F} \tilde{\Phi}_{F}\right)^{1 / 2}} .
\end{gathered}
$$

Here $\Delta_{S}$ is the superconducting order parameter of the $S$ material, $\tilde{\omega}=\omega+i H_{\text {exc }}$, where $\omega \equiv \omega_{n}=\pi T(2 n+1), \quad n= \pm 1, \pm 2, \pm 3, \ldots$ is Matsubara frequency; the summation over frequencies in (2) is cut off by the Debye frequency $\omega_{D} ; \tilde{\Phi}\left(\omega, H_{e x c}\right)=\Phi^{*}\left(\omega,-H_{\text {exc }}\right)$; the prime denotes differentiation with respect to a coordinate $x$. (Note that the momentum renormalization in the ferromagnet is not so important as the frequency renormalization.) We introduce the modified Usadel functions $\Phi_{S}=\omega F_{S} / G_{S}, \Phi_{F}=\tilde{\omega} F_{F} / G_{F}$, where $G_{F, S}$ and $F_{F, S}$ are Green's functions for the $F$ and $S$ material, respectively, to take into account the usual normalized confinement on the Green's functions $G_{F, S}$ and $F_{F, S}$ (see Refs. [21,22] for details).

The Eqs. (1)-(3) should be supplemented with the usual boundary conditions in the bulk of the $S$ metal: $\Phi_{S}(\infty)=\Delta_{S}(\infty)=\Delta_{0}(T)$, where $\Delta_{0}(T)$ is the $B C S$ value of the order parameter, and at the external surface of the $F$ metal $\Phi_{F}^{/}\left(-d_{F}\right)=0$. The boundary conditions at the $S F$ interface are [22]:

$$
\begin{gathered}
\left.\frac{1}{\tilde{\omega}} \gamma \xi G_{F}^{2} \Phi_{F}^{\prime}\right|_{x=0}=\left.\frac{1}{\omega} \xi_{S} G_{S}^{2} \Phi_{S}^{\prime}\right|_{x=0}, \\
\left.\xi \gamma_{B F} G_{F} \Phi_{F}^{\prime}\right|_{x=0}=\left.\tilde{\omega} G_{S}\left(\Phi_{S} / \omega-\Phi_{F} / \tilde{\omega}\right)\right|_{x=0},
\end{gathered}
$$

where $\gamma=\rho_{S} \xi_{S} / \rho_{F} \xi$ is a measure of the proximity effect strength, $\gamma_{B F}=R_{B} / \rho_{F} \xi$ describes the effect of the boundary transparency. Here $\rho_{S, F}$ are the normal-state resistivities of the $S$ and $F$ metals, $R_{B}$ is the product of the $S F$ boundary resistance and its area. Here and below we write our formulas for the $F$ metal using the effective coherence length of normal nonmagnetic $(N)$ metal $\xi=\left(\frac{D_{F}}{2 \pi T_{C}}\right)^{1 / 2}$ with the diffusion coefficient $D_{F}$ instead of $\xi_{F}=\left(\frac{D_{F}}{2 H_{e x c}}\right)^{1 / 2}$, to have a possibility to analyze both limits $H_{e x c} \rightarrow 0$ ( $S N$ bilayer with $\left.d_{N} \ll \xi\right)$ and $H_{e x c}>>\pi T_{C}$. The relations (1)-(5) generalize the proximity effect problem with an arbitrary interface transparency for the case of normal metal with ferromagnetic order.

\section{RESULTS}

\section{A. The spatial variation of the order parameter}

Due to a mesoscopic thickness of the $F$ metal the proximity effect problem can be reduced to the boundary problem for the $S$ layer and a relation for determining $\Phi_{F}[22,23]$. There are three parameters which enter the model: $\gamma_{M}=\gamma d_{F} / \xi$ is the measure of the strength of proximity effect between the $S$ and $F$ metals, $\gamma_{B}=\gamma_{B F} d_{F} / \xi$ describes the electrical 
quality of the $S / F$ boundary, and $H_{e x c}$ is the energy of the exchange correlation in the $F$ layer. In the general case, the problem needs self-consistent numerical solution of the Usadel equations (such mode consideration see, e.g., in Ref. [24]). Here, however, we will not discuss the quantitative calculations, but present qualitatively correct ones to consider new physics we are interested in. In this approximation, for bilayer with weak proximity effect $\gamma_{M} \ll 1$ and arbitrary value of the boundary resistance $\gamma_{B}$ (quite a realistic experimental case) for the function $\Phi_{S}$ we have the analytical solution (see Ref. [22,23]):

$$
\Phi_{S}(\omega, x)=\Delta_{0}\left\{1-\gamma_{M} \beta \tilde{\omega} \frac{\exp \left(-\beta x / \xi_{S}\right)}{\gamma_{M} \beta \tilde{\omega}+\omega A(\omega)}\right\}
$$

where $\beta^{2}=\left(\omega^{2}+\Delta_{0}^{2}\right)^{1 / 2} / \pi T_{C}$, and $A(\omega)=\left[1+\gamma_{B} \tilde{\omega}\left(\gamma_{B} \tilde{\omega}+2 \omega / \beta^{2}\right) /\left(\pi T_{C}\right)^{2}\right]^{1 / 2}$. Using $\Phi_{S}(\omega, x)(6)$ and the self-consistency condition (2) one can obtain the spatial variation of the order parameter in the $S$ layer $\Delta_{S}(x)$ for different values of $\gamma_{B}$ and $\gamma_{M} \ll 1$. As follows from the analysis, the $B C S$ value of the order parameter is reached at the distance of several $\xi_{S}$ into the $S$ layer. At fixed value of the exchange interaction $H_{e x c}$, the order parameter at $S F$ boundary $\Delta_{S}\left(0, H_{\text {exc }}\right)$ decreases with increasing boundary transparency, which leads to a decrease in the jump of the amplitude of the Cooper pairs in going from the $S$ to the $F$ layer. A decrease in the boundary transparency leads to a sharp increase of the order parameter at $S F$ boundary. The behavior on $\gamma_{M}$ and $\gamma_{B}$ parameters here is analogous to the situation in the $S N$ system (see, e.g., Ref. [21]).

Another important feature of Eq.(6) is its dependence upon $H_{\text {exc }}$. The exchange interaction influence on the spatial variation of the order parameter in the $S$ layer is shown on Fig. 1. Namely, the curves show the dependence of difference of the order parameters in the $S$ layer for the case, when magnetic interaction is turned off ( $S N$ bilayer) and the case with ferromagnetic correlation ( $S F$ bilayer) on distance from interface for fixed boundary parameters and different strength of the $F$ layer exchange field $H_{e x c}:\left[\Delta_{S}\left(x, H_{e x c}=0\right)-\Delta_{S}\left(x, H_{e x c}\right)\right]$ . It is seen that magnetic influence decreases with the increasing of the distance from $S F$ boundary and at $x>2 \xi_{S}$ the order parameter becomes equal to those for the case of $S N$ bilayer. This is not surprising, since one would expect that the induced exchange field should suppress the superconducting order parameter at some distance into the $S$ layer in excess of the $N$ layer suppression. Suppression increases with the increase of the exchange interaction parameter $H_{e x c}$ and of the proximity effect. The curves on Fig. 1 illustrate the spatial dependencies of the induced exchange correlation in the superconductor for the case of vanishing interface resistance $\gamma_{B}=0$. With an increase of the $S F$ boundary resistance the electrical coupling of the $S$ and $F$ metals decreases and in the limit $\gamma_{B} \longrightarrow \infty$ the metals become decoupled. As follows from $(6)$, far $\left(x \gg \xi_{S}\right)$ from the interface the bulk superconductivity is re-established.

\section{B. Spin-splitting of DOS and local exchange-interaction induced states}

Spin splitting of DOS in the $S$ layer is the second demonstration of a magnetism leakage into the superconductor. The Green's functions for the $S$ layer $G_{S \uparrow \uparrow}(\omega, x)$ and $G_{S \downarrow \downarrow}(\omega, x)$ for both spin subbands one can obtain using the solution (6) for the functions $\Phi_{S}(\omega, x)$ with $\tilde{\omega}=\omega+i H_{e x c}$ and $\tilde{\omega}=\omega-i H_{e x c}$, respectively. Performing the analytical continuation 
to the complex plane by the substitution $\omega \rightarrow-i \varepsilon$ we calculate the spatial dependence of quasiparticle DOS for each spin subband $N_{S \uparrow}(\varepsilon, x)=\operatorname{Re} G_{S \uparrow \uparrow}(\omega, x)$ and $N_{S \downarrow}(\varepsilon, x)=$ $R e G_{S \downarrow \downarrow}(\omega, x)$. On Fig. 2 the $N_{S \uparrow}(\varepsilon, x)$ dependence at different distances from the $S F$ interface is presented for $T<<T_{C}, H_{e x c}=5 \pi T_{C}$ and $\gamma_{M}=0.1$, and vanishing boundary resistance $\left(\gamma_{B}=0\right)$. We find that for the case of $H_{\text {exc }} \neq 0$, and $\gamma_{M} \neq 0$ the quasiparticle DOS is spin-splitted, that means $N_{S \uparrow}(\varepsilon, x) \neq N_{S \downarrow}(\varepsilon, x)$ and $N_{S \uparrow}(\varepsilon, x) \neq N_{S \uparrow}(-\varepsilon, x)$, in the $S$ layer at the distance of a few $\xi_{S}$ from the $S F$ boundary. As one can expect from the fermionic symmetry, the spin-up particles and spin-down holes have the same DOS, and likewise for spin-down particles and spin-up holes. The spin-splitting decreases with an increase of the distance from the boundary and vanishes in the bulk of the $S$ layer (see curve 4 on Fig. 2). The DOS of Cooper's pairs $\aleph_{\uparrow}(\varepsilon, x)=R e F_{S \uparrow \downarrow}(\omega, x), \aleph_{\downarrow}(\varepsilon, x)=R e F_{S \downarrow \uparrow}(\omega, x)$ is spin-splitted, too. These features are due to the initial spin-splitting electrons on the Fermi surface in the $F$ metal and characterize the $S F$ bilayer as a united system.

On Fig. 3 the $N_{S \uparrow}(\varepsilon, x)$ dependence is presented for $x / \xi_{S}=1$ and different values of the exchange energy for bilayer with finite boundary transparency $\gamma_{B}=0.1$ and proximity effect strength $\gamma_{M}=0.1$. The behavior on $\gamma_{B}$ parameter is due to two mechanisms: with decreasing boundary transparency spin-splitting increases if $\gamma_{B}<0.2$, however, for large enough $\gamma_{B}>0.2$ the decrease of the interface electrical quality preponderates and DOS approaches the BCS value.

Other important features, shown on Figs. 2 and 3, are the local states that exist inside the energy gap at the distances of a few $\xi_{S}$ from the $S F$ boundary. These subgap states are absent at the $S F$ boundary $(x=0)$, far from the $S F$ interface, and also absent if $H_{\text {exc }}=0$. For small values of $\gamma_{M}$ and $\gamma_{B}=0$, as follows from Eq.(2) and solution (6), $N_{S \uparrow}(\varepsilon, x)$ has singularities for

$$
\varepsilon= \pm \Delta_{0}\left\{1-\frac{\gamma_{M} \beta_{\varepsilon} \tilde{\varepsilon}}{\varepsilon+\gamma_{M} \beta_{\varepsilon} \tilde{\varepsilon}} \exp \left(-\beta_{\varepsilon} x / \xi_{S}\right)\right\}
$$

where $\beta_{\varepsilon}^{2}=\left(\Delta_{0}^{2}-\varepsilon^{2}\right)^{1 / 2} / \pi T_{C}$ and $\tilde{\varepsilon}=\varepsilon-H_{e x c}$. We found the singularities inside the superconducting gap, $-\Delta_{0}<\varepsilon<\Delta_{0}$, by numerical calculations. The solutions of Eq.7 for $x / \xi_{S}=0,1,5$ and for $\gamma_{M}=0.1, H_{e x c}=5 \pi T_{C}$ are presented on Fig.4 in the diagram form. The local states are thus definitely not due to the spatial variation of the pair potential, but due to Cooper pairs breaking in the superconductor by the exchange-induced magnetic correlation. The region of their existence increases with the increasing of $H_{\text {exc }}$, or increasing pair breaking effects. In the absence of spin-flip (e.g., spin-orbit) scattering, the subgap bands accommodate only one spin of quasiparticles. These bands bear superficial resemblance to both the bands observed at interface of superconductor and perfectly insulating ferromagnet [25] and bulk superconductor containing finite concentrations of magnetic impurities $[26,27]$.

\section{Local magnetization}

As was mentioned above, the influence of the ferromagnet on the superconductor is reflected in a nonzero value of the difference in the densities of states for spin-up and spin-down electrons, $N_{S \uparrow}(\varepsilon, x)$ and $N_{S \downarrow}(\varepsilon, x)$. This DOS splitting causes inhomogeneous quasiparticle 
spin density in the $S$ metal, i.e., an effective magnetization $M_{S}(x)$ of the $S$ layer, that can be found using the relation (see, e.g., [28]) :

$$
M_{S}(x)=M_{O} \int_{0}^{\infty} d \varepsilon\left\{N_{S \uparrow}(\varepsilon, x)-N_{S \downarrow}(\varepsilon, x)\right\} f(\varepsilon)
$$

where $M_{O}=g S_{e} \mu_{B}\left(=\mu_{B}\right), S_{e}=1 / 2, g=2$ and $f(\varepsilon)=1 /[\exp (\varepsilon / T)+1]$ is Fermi distribution function. This is confirmed by numerical calculations of $M_{S}(x)$ Eq.(8) shown in Figs. 5-7. Fig. 5 shows the leakage of magnetization into the superconductor versus distance from the $S F$ interface for fixed boundary parameters and different exchange field energy values. Fig. 6 shows the same magnetic characteristics but for $S F$ sandwich with fixed exchange energy and boundary transparency, and different proximity effect strength. The curves on Fig. 7 illustrate inhomogeneous magnetism induced into the superconductor by proximity effect, $M_{S}(x)$, for the $S F$ bilayers with different interface transparency and fixed $H_{e x c}$ and $\gamma_{M}$. Following DOS dependance, the magnetization behavior on $\gamma_{B}$ parameter is due to two mechanisms: firstly, $\gamma_{B}<0.2$, with decreasing boundary transparency spinsplitting increases and $M_{S}(x)$ increases too, secondly however, for large enough $\gamma_{B}>0.2$ the decreasing of the interface electrical quality preponderates and induced magnetization decreases.

All the curves of $M_{S}(x)$ show essentially non-linear behavior versus distance from the $S F$ interface, and have important common feature - a damped oscillatory behavior on a scale of superconducting coherence length $\xi_{S}$. As is evident from the plots on Figs. 5-7, induced by proximity effect magnetic correlations in the $S$ layer are inhomogeneous in character with spatially dependent magnitude and direction of its molecular field.

\section{CONCLUSION}

We have calculated the magnetic proximity effect induced in the superconductor at $S F$ interface. We find that an equilibrium exchange of electrons between the $F$ and $S$ metals results not only in proximity induced inhomogeneous superconductivity of the $F$ metal, as was found earlier, but in proximity induced inhomogeneous magnetism of the $S$ metal, too. Our results show that the appearing damping magnetic correlations in the superconductor are quite robust and, to our opinion, are quite possible for experimental measurements.

Experimental studies of magnetic proximity effect in $S F$ structures are nowadays unclear and controversial. Tedrow et. al. [29] were the first who carried out high-field tunneling measurements of the quasiparticle DOS in superconducting films of $A l$ backed by $E u O$, which in bulk form is a ferromagnetic insulator with a Curie temperature $T_{C R}=70 \mathrm{~K}$. The tunnel conductances of these junctions show a spin splitting of the $A l$ DOS in excess of the splitting observed for $A l$ films in contacts with nonmagnetic substrates in equivalent magnetic field. The authors interpreted the excess spin splitting in terms of a coupling between the $A l$ conduction electrons and the $E u O$ magnetization. However, Tokuyasu et.al. [30] argued that the observed excess Zeeman splitting in EuO/Al proximity contacts cannot be explained in such a mode, and considered that excess splitting is the reflection of the exchange coupling of the tunneling electrons with $E u^{2+}$ spins at the interface. Recent experiments $[31,15]$ revealed that the effective thickness of the magnetic layer in $S F$ hybrid structures usually is much larger than its physical thickness. The increase of the thicknesses 
is so great that in all samples, except for those with extremely thin magnetic layers, the crossover to a 3D state superconductivity is never in fact observed experimentally. This is to be contrasted with the case of nonmagnetic spacer layers, where these two length scales are comparable. Taking into account our results, we explain that the rise of the effective magnetic layer thickness in the $S F$ sandwiches is due to magnetic proximity effect. Namely, the induced magnetic correlation into the $S$ layer depletes Cooper's pair density at the $S F$ boundary that results in an excess thickness of the magnetic layer.

In conclusion, we study the magnetic correlations into a superconductor that have been induced at $S F$ interface due to proximity effect. Equilibrium leakage of magnetism into the $S$ metal results in (i) additional spatial suppression of the order parameter in the $S$ layer, (ii) spatial spin splitting of the quasipaticles DOS, (iii) local bands that appear inside the energy gap in the $S$ layer, and (iv) spatially dependent magnitude and direction induced magnetization of the $S$ layer. The existence of these magnetic properties of the $S$ metal is

quite important for $S F$ nanostructures and should be taken into account while comparing theoretical results with experimental data.

\section{ACKNOWLEDGMENT}

The authors are grateful to V. V. Ryazanov for useful discussions. 


\section{REFERENCES}

[1] P. Fulde and A. Ferrel, Phys. Rev. 135, A550 (1964).

[2] A. I. Larkin, Yu. N. Ovchinnikov, Sov. Phys. JETP 20, 762 (1965).

[3] E.A.Demler, G.B.Arnold, M.R.Beasley. Phys.Rev. B 55, 15174 (1997).

[4] A. I. Buzdin, L. N. Bulaevskii, and S. V. Panyukov, Sov. Phys. JETP Lett. 35, 178 (1982)

[5] A. I. Buzdin, M. Yu. Kupriyanov, Sov.Phys.JETP Lett. 53, 321 (1991).

[6] F. S. Bergeret, A. F. Volkov, and K. B. Efetov, Phys.Rev.Lett. 86, 3140 (2001).

[7] V. N. Krivoruchko and E.A. Koshina, Phys.Rev. B 64, 172511 (2001).

[8] H. K. Wong, B. Y. Jin, H. Q. Yang, J.B.Ketterson and J.E.Hilliard, J. Low Temp. Phys.63, 307 (1986).

[9] Z. Radović, M. Ledvij, L. Dobrosavljević-Grujić, A. I. Buzdin and J. M. Clem, Phys. Rev. B 44, 759 (1991).

[10] V. V. Ryazanov, V. A. Oboznov, A. Yu. Rusanov, A. V. Veretennikov, A.A.Golubov, and J. Aarts, Phys. Rev. Lett. 86, 2427 (2001).

[11] V. V. Ryazanov, V. A. Oboznov, A. V. Veretennikov, and A. Yu. Rusanov, Phys. Rev. B 65, 020501(R) (2002).

[12] T. Kontos, M. Aprili, J. Lesueur, F. Genet, B. Stephanidis and R. Boursier, condmat/0201104 (unpablished).

[13] T. Kontos, M. Aprili, J. Lesueur, and X. Grison, Phys. Rev. Lett. 86, 304 (2001).

[14] J. S. Jiang, D. Davidović, D. H. Reich, and C. L. Chien, Phys. Rev. Lett. 74, 314 (1995); C. L. Chien, J. S. Jiang, J. Q. Xiao, D. Davidović, D. H. Reich. J. Appl. Phys. 81, 5358 (1997).

[15] Y. Obi, H. Wakou, M. Ikebe, and H. Fujimori, Czech.J.Phys. 46, 731 (1996).

[16] Th. Mühge, N. N. Garif'yanov, Yu. V. Guryunov, G. G. Khaliulin, L.R.Tagirov, K. Westerholt, I. A. Garifullin, and H. Zabel,. Phys. Rev. Lett. 77, 1857 (1996).; Th. Mühge, K. Westerholt, H. Zabel, N. N. Garif'yanov, Yu. V. Guryunov, I. A. Garifullin, and G. G. Khaliulin. Phys.Rev. B 55, 8945 (1997).

[17] C. Strunk, C. Sürgegers, U.Paschen, and H. v. Löhneysen, Phys. Rev. B 49, 4053 (1994).

[18] J. Aarts, J. M. E. Geers, E. Brück, A. A. Golubov, and R. Coehoorn. Phys. Rev. B 56, 2779 (1997).

[19] S. Oh, Y.-H. Kim, D.Youm, and M.R. Bearsley, Phys. Rev. B 63, 052501 (2000).

[20] K. Usadel, Phys. Rev. Lett. 25, 560 (1970).

[21] A. A. Golubov, E. P. Houwman, J. G. Gijsbertsen, V. M. Krasnov, J. Flokstra, H. Rogalla, and M. Yu.Kuprijanov, Phys. Rev. B 51, 1073 (1995); M. Yu. Kuprianov, V. F. Lukichev, Sov. J. Low Temp. Phys. 8, 1045 (1982).

[22] E. A. Koshina, V. N. Krivoruchko. Low Temp. Phys. 26, 115 (2000) [Low.Tem.Fiz. 26, $157(2000)]$.

[23] E. A. Koshina and V. N. Krivoruchko. Phys.Rev. B 63, 224515 (2001).

[24] G. Brammertz, A. Poelaert, A. A. Gulubov, P. Verhoeve, A. Peacock, and H. Rogalla, J. Appl. Phys. 90, 355 (2001); A. A. Golubov, M. Yu. Kupriyanov, and Ya. V. Fominov, cond-mat/0201249 (unpablished).

[25] M. J. DeWeert, G. B. Arnold, Phys. Rev. B 39, 11307 (1989).

[26] H. Shiba, Prog. Theor. Phys. 40, 435 (1968).

[27] A. J. Rusinov, Sov. Phys. JETP Lett. 9, 85 (1969). 
[28] N. W. Ashcroft, N. D. Mermin, Solid State Physics. 1976 by Holt, Rinehart and Winston. Chapt. 31.

[29] P. M. Tedrow, J. E. Tkaczyk, and A. Kumar, Phys. Rev. Lett. 56, 1746 (1986).

[30] T. Tokuyasu, J. A. Sauls, and D. Rainer, Phys. Rev. B 38, 8823 (1988).

[31] F. Y. Ogrin, S. L. Lee, A. D. Hillier, A. Mitchell, and T.-H.Shen, Phys. Rev. B 62, 6021 (2000).

\section{Figure Captures}

Fig. 1. The difference of the superconducting order parameter in the $S$ layer versus distance from the interface for $S N$ and $S F$ structures with the same boundary parameters $\left(\gamma_{M}=0.1, \gamma_{B}=0\right)$, and different ferromagnetic field energy $H_{\text {exc }} / \pi T_{C}=8,9,10,12$ and 15.

Fig. 2. Normalized density of state for spin "up" quasiparticles in the $S$ layer of the $S F$ sandwich for $\gamma_{M}=0.1, \gamma_{B}=0$ and $H_{e x c}=5 \pi T_{C}$, and various distances from the $S F$ interface: $x / \xi_{S}=0,1,5$, and 30 (curves $1,2,3$, and 4 , respectively).

Fig. 3. Same as on Fig. 2 for $\gamma_{M}=0.1, \gamma_{B}=0.1$ and $x=\xi_{S}$, and various ferromagnetic field energies: $H_{e x c} / \pi T_{C}=1,2$, and 5 (curves 1,2 , and 3 , respectively).

Fig. 4. Diagram solutions of the Eq.7 for $\gamma_{M}=0.1, H_{e x c}=5 \pi T_{C}$ and $x / \xi_{S}=0,1,5$, (curves 1,2, and 3, respectively). The regions where the local states exist are shaded; see also Fig. 2 for comparison.

Fig. 5. Leakage of magnetization into the $S$ material versus distance from the interface for $S F$ sandwich for $\gamma_{M}=0.1, \gamma_{B}=0$, and different exchange energies $H_{e x c} / \pi T_{C}=7,5$, and 3 (curves 1,2 , and 3 , respectively).

Fig. 6. Same as on Fig. 4 for $\gamma_{B}=0, H_{\text {exc }}=3.5 \pi T_{C}$ and different proximity effect strength $\gamma_{M}=0.1,0.15,0.2$ (curves 1, 2, and 3, respectively).

Fig. 7. Same as on Fig. 4 for $\gamma_{M}=0.1, H_{e x c}=5 \pi T_{C}$ and different $S F$ boundary transparency $\gamma_{B}=0.0,0.05$, and 0.1 (curves 1,2 , and 3 , respectively). 


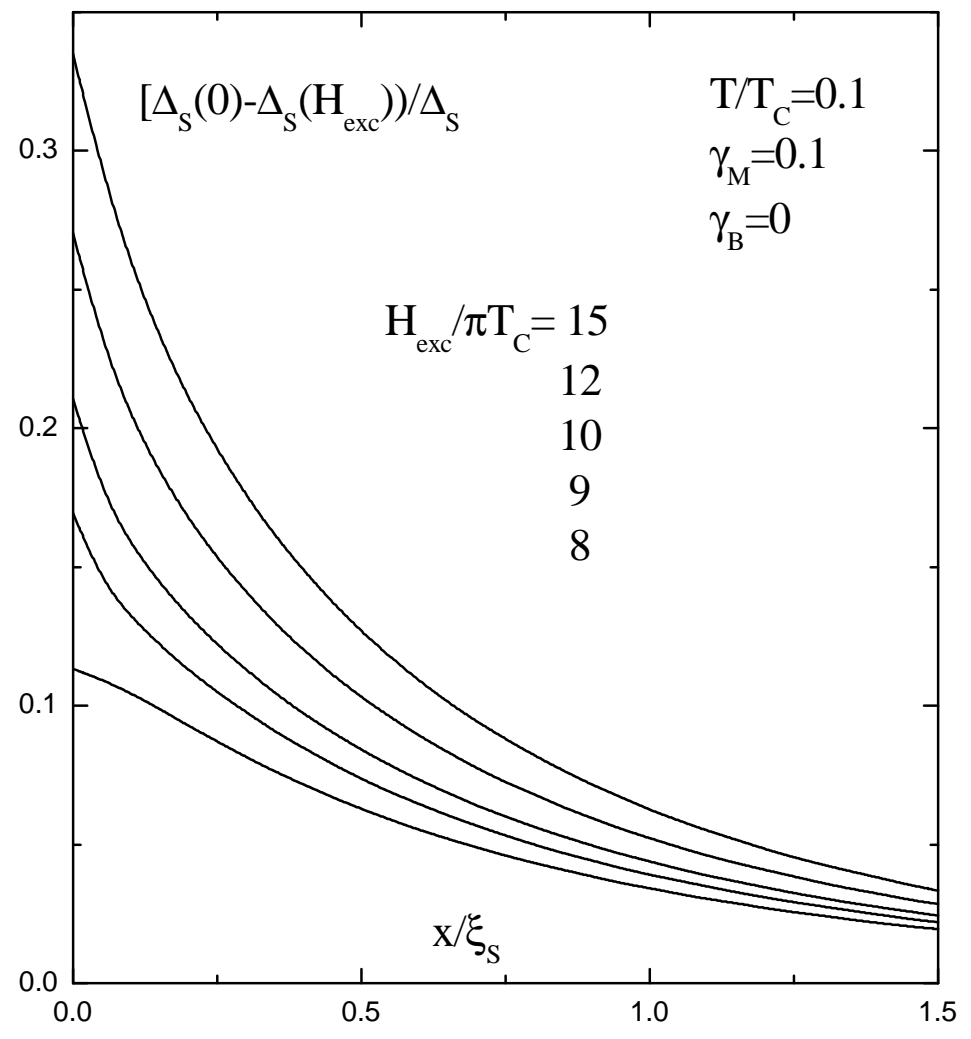

Fig.1 


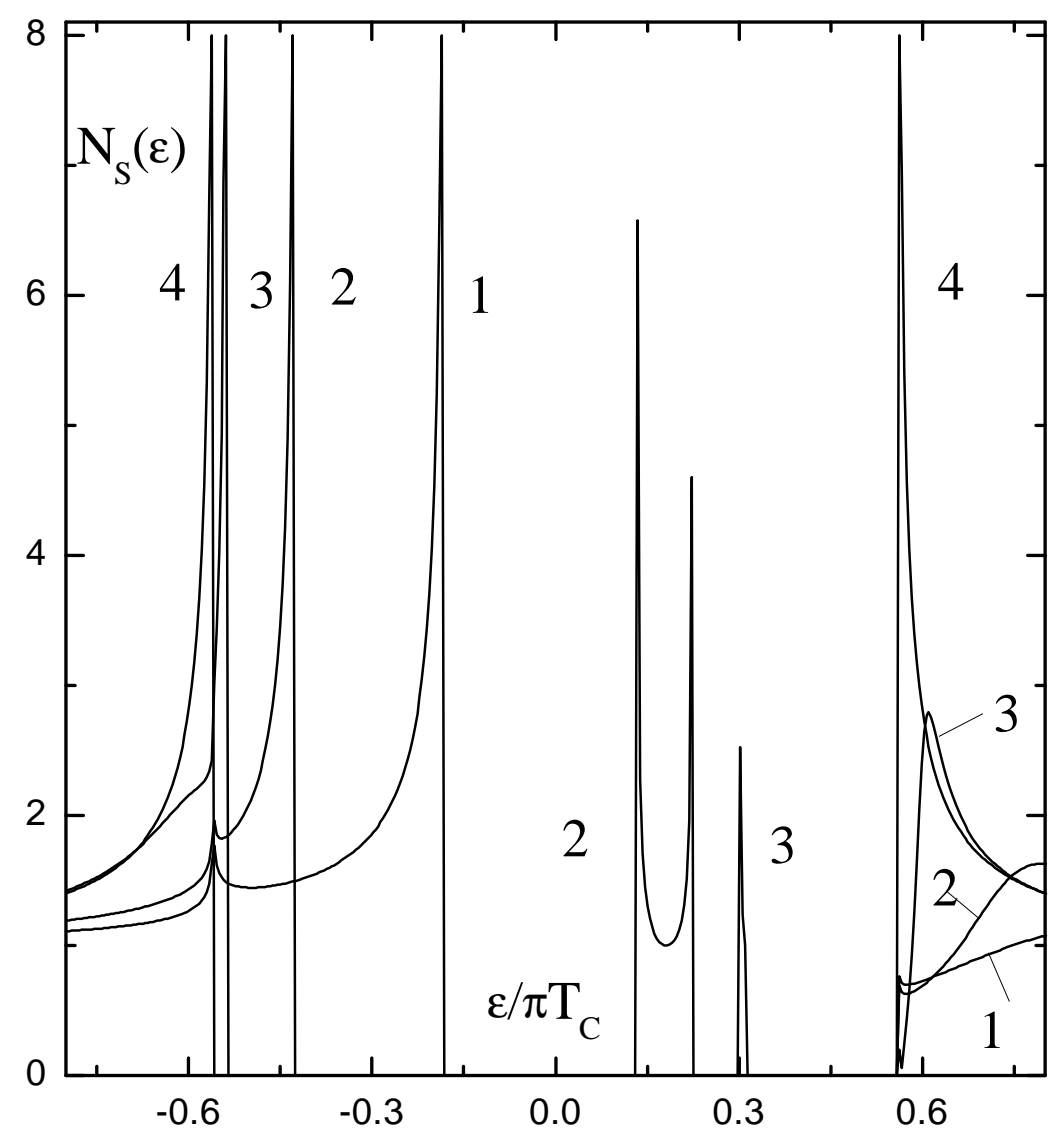

Fig.2 


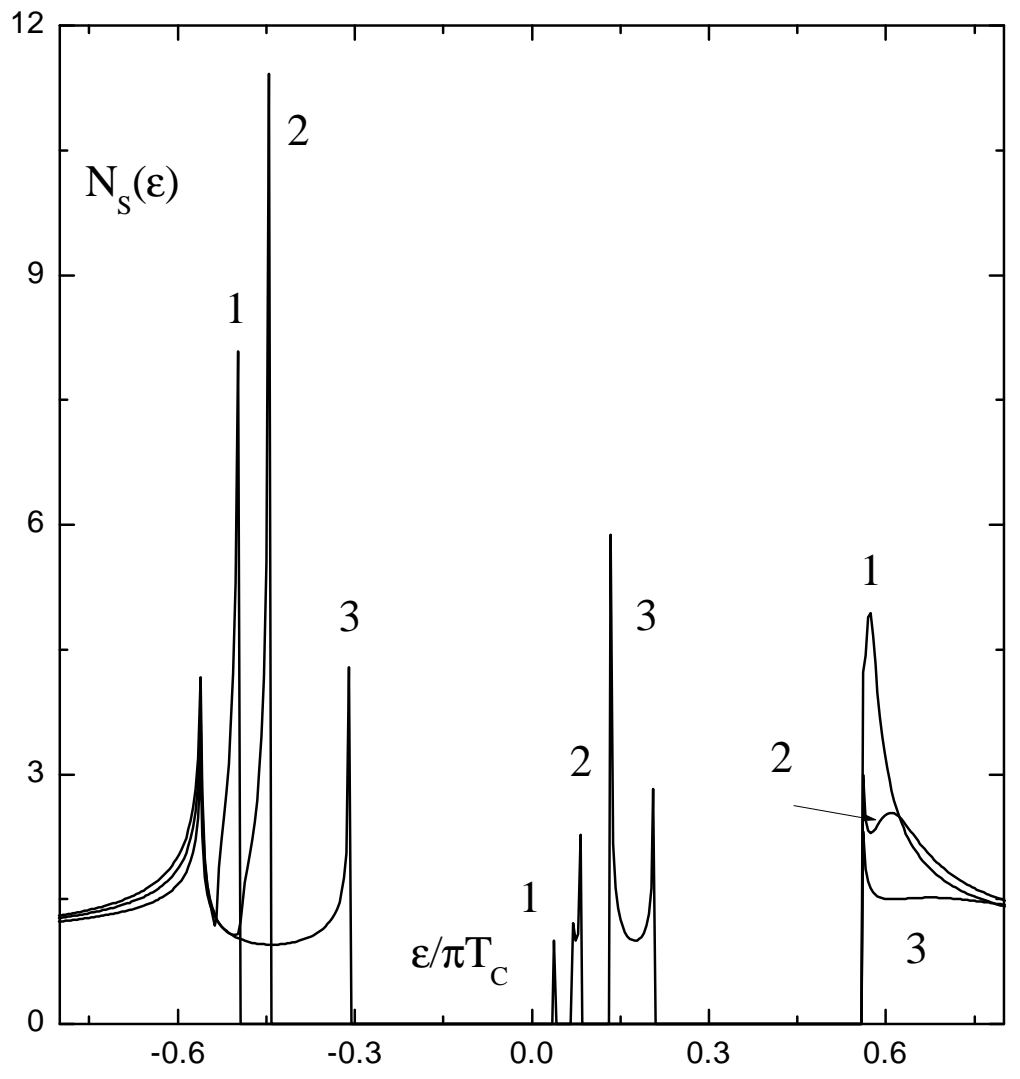

Fig. 3 


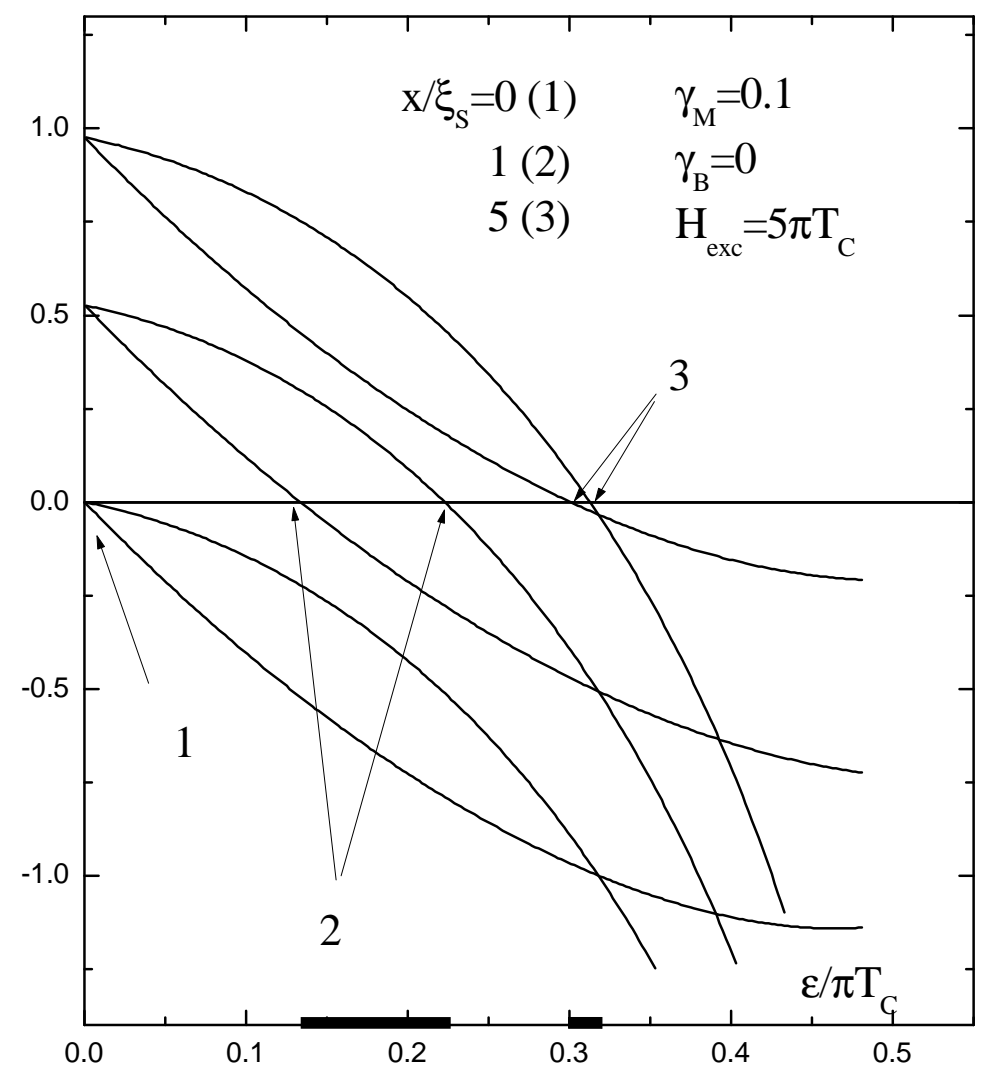

Fig. 4 


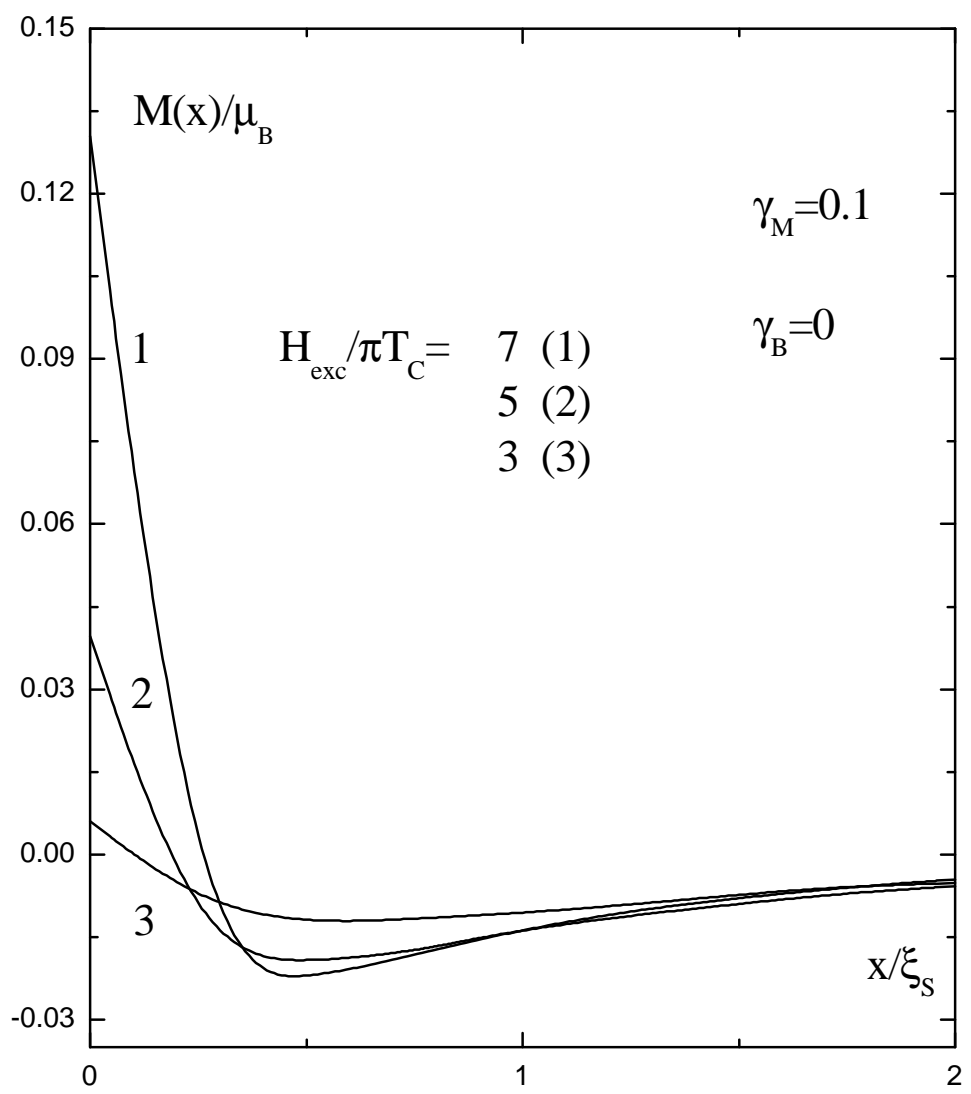

Fig.5 


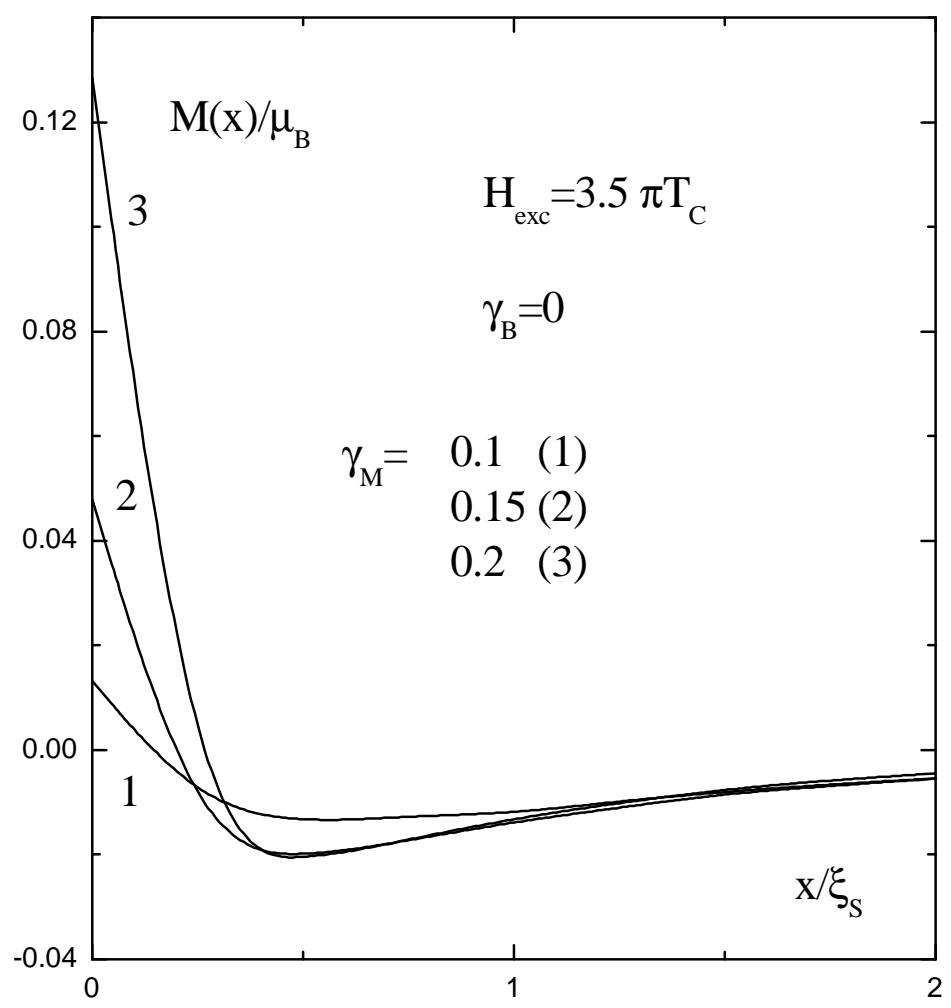

Fig.6 


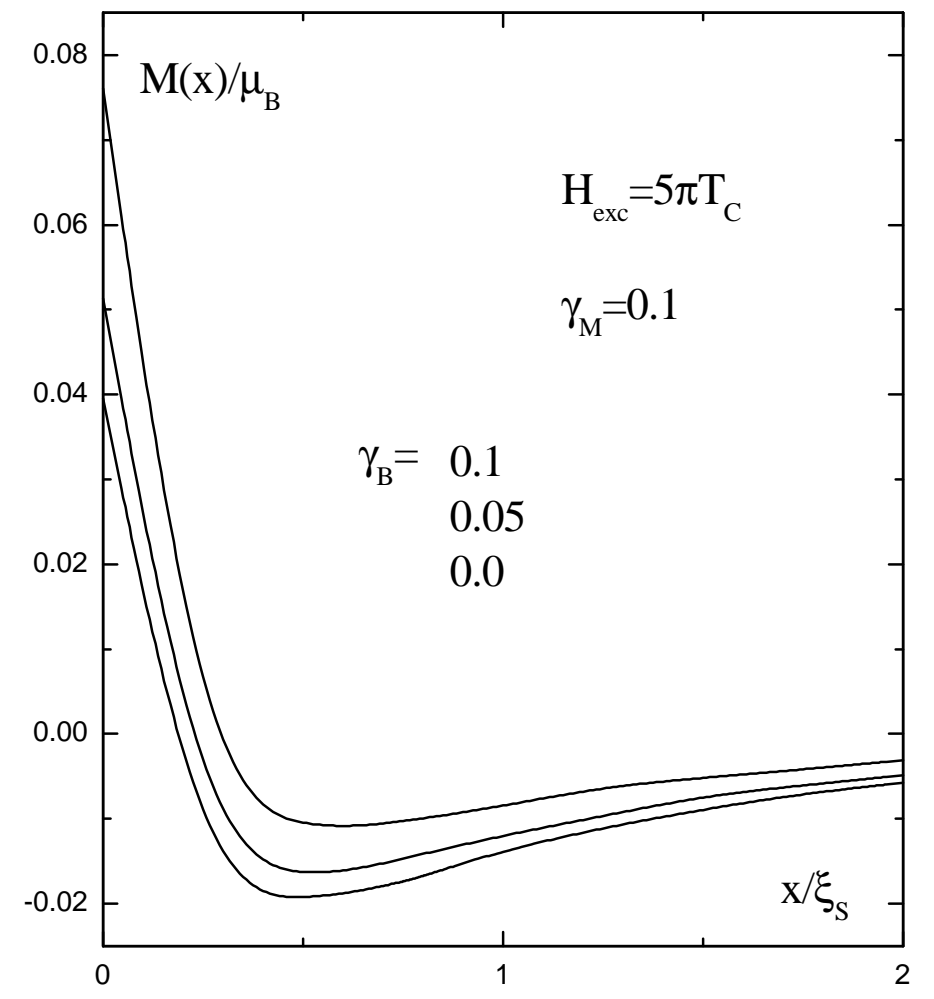

Fig.7 